\title{
Erratum: Engineering Matter Interactions Using Squeezed Vacuum [Phys. Rev. X 7, 021041 (2017)]
}

\author{
Sina Zeytinoğlu@, Ataç İmamoğlu, and Sebastian Huber
}

(Received 31 January 2019; published 20 December 2019)

DOI: 10.1103/PhysRevX.9.049903

We report two errors in our manuscript. First, we note that the Hamiltonian in Eq. (4) of the manuscript assumes that the squeezed vacuum modes evolve under a two-photon driven Hamiltonian in the frame rotating with half the optical parametric amplifier (OPA) pump frequency

$$
H_{\mathrm{vac}}^{s} / \hbar=\omega a^{\dagger} a+i \frac{\epsilon}{2}\left[a^{2}-\left(a^{\dagger}\right)^{2}\right]
$$

which, contrary to the setup depicted in Fig. 1(a) of the manuscript, implies that the emitters are placed within the OPA cavity. While all the calculations from this starting point are valid, the spatial control of the dipole-dipole interactions require a different experimental setup, which we depict in Fig. 1 below.

We emphasize that, for the single-mode model in Eq. (1) to be valid, we require the coupling between the nonlinear mode of the $\chi^{(2)}$ material and the cavity modes to be much smaller than the cavity-free-spectral range. In the experimental setup depicted in Fig. 1, the spatial control of the dipole-dipole interactions can be achieved by shaping the mode structure of the laser drive, which is necessary for the Raman scheme discussed in our manuscript. Using the single-cavity mode also entails that all expressions involving summations over different modes $l$ should be replaced by single-mode expressions and that the cavity mode transforms according to $a \rightarrow u a+v a^{\dagger}$ under the squeezing transformation. Here, the parameters are $u=\cos (s)$ and $v=\sin (s) e^{i \vartheta_{s}}$, where $s=\frac{1}{4} \log [(\omega+\epsilon) /(\omega-\epsilon)]$ and $\vartheta_{s}$ is the squeezing angle.

When the cavity decay rate is negligible, an intracavity squeezed vacuum can be prepared by tuning the external parameter $\epsilon(t)$ at a rate faster than the cavity decay rate while ensuring adiabaticity with respect to the Hamiltonian dynamics. In this scenario, the interaction between the emitters and the OPA mode is enhanced as discussed in the original proposal. By virtue of being placed already in a cavity, the single-atom cooperativity that is reported in the manuscript can be easily achieved. In the presence of a nonzero cavity decay, the interaction strength remains enhanced, but the squeezed OPA mode acquires a thermal occupation in the steady state [1]. In the dispersive regime, the photon number fluctuations associated with the thermal occupation cause dephasing of the emitters, as discussed in Ref. [2].

Additionally, in the second and the fourth terms of the expression below Eq. (D3), the function $\Xi(r)$ is incorrectly complex conjugated. This typo is carried over to the definition of $\tau$ in Eq. (D4). For a single mode, the correct definition is $\tau^{\dagger}(r) \equiv \cos (\kappa) \Xi(r) O^{\dagger}(r)-\sin (\kappa) e^{-i \vartheta_{s}} \Xi^{*}(r) O(r)$, where $\Xi(r)$ is the mode function associated with the cavity-assisted Raman transition. This correction then leads to the following approximate interaction Hamiltonian:

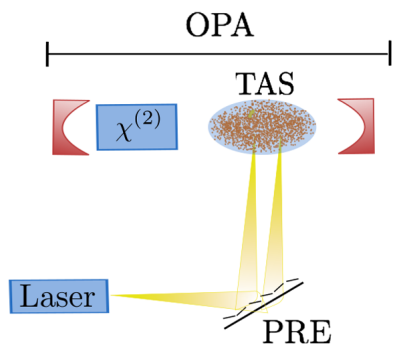

FIG. 1. The sketch of the experimental setup consistent with Eq. (1) of the manuscript. The trapped atomic system (TAS) is now placed within the OPA cavity, and the spatial control of the dipole-dipole interactions between the atoms is achieved by manipulating the spatial features of the laser drive (which is necessary for the proposed Raman scheme) using programmable reflective elements (PRE).

Published by the American Physical Society under the terms of the Creative Commons Attribution 4.0 International license. Further distribution of this work must maintain attribution to the author(s) and the published articles title, journal citation, and DOI. 


$$
\begin{aligned}
H_{\mathrm{int}}\left(r, r^{\prime}\right) \approx & \Xi(r) \Xi\left(r^{\prime}\right)\left(|u|^{2}+|v|^{2}\right) \frac{\eta^{2}}{\omega}\left\{\hat{O}^{\dagger}(r) \hat{O}\left(r^{\prime}\right)+\hat{O}^{\dagger}\left(r^{\prime}\right) \hat{O}(r)\right. \\
& \left.-2 \cos (\kappa) \sin (\kappa)\left[\exp \left(i \vartheta_{s}\right) \hat{O}^{\dagger}(r) \hat{O}^{\dagger}\left(r^{\prime}\right)+\exp \left(-i \vartheta_{s}\right) \hat{O}\left(r^{\prime}\right) \hat{O}(r)\right]\right\}
\end{aligned}
$$

assuming that the dipole transition frequency is much smaller than $\omega$ (as in the manuscript) and $\arg \left[\Xi_{l}(r)\right]=\arg \left[\Xi_{l}\left(r^{\prime}\right)\right]=0$. As a result, contrary to our claim, the terms proportional to $\hat{O} \hat{O}$ and $\hat{O}^{\dagger} \hat{O}^{\dagger}$ cannot be eliminated by tuning the squeezing angle $\vartheta_{s}$. However, our conclusion that the enhancement of interactions is reduced by half holds when the rotating wave approximation is valid in the squeezed frame. We note that the enhancement of interactions due to a squeezed vacuum in the case of Jaynes-Cummings interactions was studied by Refs. [3,4] following our publication.

[1] M.-A. Lemonde, N. Didier, and A. A. Clerk, Enhanced Nonlinear Interactions in Quantum Optomechanics via Mechanical Amplification, Nat. Commun. 7, 11338 (2016).

[2] J. Gambetta, A. Blais, D. I. Schuster, A. Wallraff, L. Frunzio, J. Majer, M. H. Devoret, S. M. Girvin, and R. J. Schoelkopf, QubitPhoton Interactions in a Cavity: Measurement-Induced Dephasing and Number Splitting, Phys. Rev. A 74, 042318 (2006).

[3] W. Qin, A. Miranowicz, P.-B. Li, X.-Y. Lü, J. Q. You, and F. Nori, Exponentially Enhanced Light-Matter Interaction, Cooperativities, and Steady-State Entanglement Using Parametric Amplification, Phys. Rev. Lett. 120, 093601 (2018).

[4] C. Leroux, L. C. G. Govia, and A. A. Clerk, Enhancing Cavity Quantum Electrodynamics via Antisqueezing: Synthetic Ultrastrong Coupling, Phys. Rev. Lett. 120, 093602 (2018). 